\title{
THE LINEARIZATION OF A BOUNDARY VALUE PROBLEM FOR A SCALAR CONSERVATION LAW*
}

\author{
FILIPA CAETANO ${ }^{\dagger}$
}

\begin{abstract}
The aim of this paper is to study a boundary value problem for a linear scalar equation with discontinuous coefficients. This kind of problem appears in the framework of the analysis of the linearized stability of a fluid flow with respect to small perturbations of the boundary data. The linear equation that we are interested in is obtained by linearizing the equations which govern the flow, and involves discontinuous coefficients and nontrivial products. We present a direct approach based on the one introduced by Godlewski and Raviart, which leads to measure solutions, gives a sense of these nontrivial products, and yields simple numerical schemes that give good results.
\end{abstract}

Key words. Scalar conservation laws, linearization, boundary value problem, shocks, measure solutions.

AMS subject classifications. 35L65, 35L50, 35R05, 76E99

\section{Introduction}

The stability or instability of fluid flows with respect to perturbations of the data is an important problem which is encountered in number of applications. In general, these fluid flows are modeled by nonlinear systems of conservation laws and the study of the nonlinear stability is a difficult problem. The first step to deal with this difficulty consists of studying the linearized stability, i.e., the evolution in time of the solution of the linear system obtained by linearizing the nonlinear system around a given flow, called the basic solution. If the basic solution is discontinuous, the linearized equation has discontinuous coefficients and does not possess in general a solution in any functional space.

The classical approach for studying the problem of linearized stability was developed by Majda (cf. $[10,11]$ ) and it consists of linearizing the nonlinear system in the domains where the basic solution is smooth and linearizing the Rankine-Hugoniot jump condition at the discontinuity points. However, this approach leads to a multidimensional system and is not easy to deal with in the context of numerical simulations. In [6] and [8], the authors proposed a "direct approach" for the linearized equation, in the setting of the Cauchy problem, which leads to a solution in the class of measures. Indeed, this solution consists of the sum of a function and a measure carried by the curves of discontinuity of the basic solution. In [3] and [4], Bouchut and James proved, in the framework of duality solutions, that this solution can be obtained as the derivative of the BV solution of the nonlinear equation written in non-conservative form. Also in [2], Bardos and Pironneau presented a method to differentiate, with respect to a parameter, equations in divergence form whose solutions present discontinuities. In [12], Poupaud and Rascle used another approach, based on the notion of generalized Filippov characteristics, to study the Cauchy problem for the multi-dimensional transport equation. Concerning the numerical approximation of the linearized equation, in [9] Gosse and James proved the convergence of a class of numerical schemes towards the duality solution of the Cauchy problem for the linearized equation.

\footnotetext{
*Received: October 27, 2007; accepted (in revised version): February 22, 2008. Communicated by Francois Bouchut.

${ }^{\dagger}$ UPMC Univ Paris 06, UMR 7598, Laboratoire Jacques-Louis Lions, F-75005, Paris, France and Centro de Matemática e Aplicações, FCT-UNL (caetano@ann.jussieu.fr).
} 
In this paper, we follow the direct approach introduced in [8] to analyze the linearized boundary value problem. For the nonlinear boundary value problem for a scalar conservation law, in [1] Bardos, Le Roux et Nédélec proved the existence of a solution that satisfies an appropriate boundary condition, which cannot be used in a classical sense, by using a vanishing viscosity method. We consider here the solution given by [1] and we study the linear boundary value problem obtained by linearizing the nonlinear equation at a given discontinuity solution, with respect to perturbations of the boundary data.

The aim of the paper is to prove that, on the one hand, in the case of a boundary value problem we still obtain a measure solution carried by the discontinuities of the basic solution and, on the other hand, that when the non linear flux is a convex function, the formulation of the boundary condition that we propose for the linearized problem can be obtained by linearizing the boundary condition proposed by Bardos, Le Roux and Nédélec. Furthermore, we adapt a Roe type numerical scheme, proposed in [6], to the boundary value problem and present some numerical results.

The plan of the paper is as follows. In section 2 we recall the results of Bardos, Le Roux and Nédélec concerning the nonlinear boundary value problem for scalar conservation laws and introduce the formalism to deduce the linearized problem. In section 3 we solve the linearized problem by a direct approach, when the basic solution is discontinuous along a shock curve. Our main result gives an explicit solution for the linearized boundary value problem when the boundary data is a Dirac mass at a point that corresponds to the time when the discontinuity develops. This kind of measure data appears when we perturb the initial "position" of the data discontinuity. In section 4, we put ourselves in the setting of a convex flux function. We prove that, when the boundary is not characteristic, the linearization of the boundary condition given in [1] by Bardos, Le Roux and Nédélec, yields a boundary condition for the linearized problem which is coherent with its linear structure. Finally, in section 5, we adapt the linearized numerical scheme of Roe type proposed in [6] to the boundary value problem. We present some tests concerning Burgers' equation and give some numerical results concerning the error committed by using this approach.

\section{The linearized scalar conservation law with boundary condition}

Consider the initial and boundary condition problem for a scalar conservation law in the quarter of plane $\{(x, t): x \geq 0, t \geq 0\}$

$$
\begin{cases}u_{t}+(f(u))_{x}=0, & x>0, t>0, \\ u(x, 0)=u_{0}(x), & x>0, \\ u(0, t)=a(t), & t>0,\end{cases}
$$

with given initial data $u_{0} \in B V\left(\left[0,+\infty[)\right.\right.$, boundary data $a \in B V\left(\left[0,+\infty[)\right.\right.$ and $C^{1}$ flux function $f$. It is well known that boundary condition $u(0, t)=a(t)$ can not in general be imposed in a strong sense. In [1], Bardos, Le Roux and Nédélec reformulated this condition by requiring $u(0, t)$ to satisfy, for all $t>0$,

$$
\frac{f(u(0, t))-f(k)}{u(0, t)-k} \leq 0, \forall k \text { between } u(0, t) \text { and } a(t)
$$

The authors proved that problem (2.1) is well-posed and that the obtained solution belongs to $B V([0,+\infty[\times[0, T[), \forall T>0$, with the boundary condition characterized by (2.2). 
An equivalent way to formulate the boundary value problem for a scalar equation consists of introducing, for $t>0$, the set

$$
\mathcal{O}(a(t))=\left\{w\left(0^{+} ; a(t), u_{r}\right), u_{r} \in \mathbb{R}\right\},
$$

where $w\left(\frac{x}{t} ; u_{l}, u_{r}\right)$ is the solution of the Riemann problem

$$
\left\{\begin{array}{l}
u_{t}+(f(u))_{x}=0, x \in \mathbb{R}, \quad t>0, \\
u(x, 0)=u_{0}(x)= \begin{cases}u_{l}, & x<0, \\
u_{r}, & x>0 .\end{cases}
\end{array}\right.
$$

Then, an equivalent condition to $(2.2)$ consists of requiring $u(0, t)$ to satisfy $u(0, t) \in$ $\mathcal{O}(a(t))$, for all $t>0$ (see [5]).

Let $u=u(x, t)$ be a solution of problem (2.1), which we will refer to as the basic solution. We put ourselves in the context of analyzing the linearized stability of $u$ with respect to perturbations of the boundary data $a$. Let us introduce $a_{\varepsilon}=a+\varepsilon b_{\varepsilon}+o(\varepsilon)$, a small perturbation of the boundary data $a$. Assume that the perturbed solution $u_{\varepsilon}$ depends smoothly on the parameter $\varepsilon$ and that it can be developed around $u$ as $u_{\varepsilon}=u+\varepsilon v_{\varepsilon}+o(\varepsilon)$ (we do not justify here this expansion). Then we obtain, at least formally, that the first order perturbation $v=\lim _{\varepsilon \rightarrow 0} \frac{u_{\varepsilon}-u}{\varepsilon}$ satisfies the linearized problem

$$
\begin{cases}v_{t}+\left(f^{\prime}(u) v\right)_{x}=0, & x>0, t>0, \\ v(x, 0)=v_{0}(x), & x>0, \\ v(0, t)=b(t), & t>0,\end{cases}
$$

where $b=\lim _{\varepsilon \rightarrow 0} \frac{a_{\varepsilon}-a}{\varepsilon}$, which is obtained by linearizing problem (2.1) around the basic solution $u$ (we have in fact $v_{0}=0$, but we consider here in a general setting an appropriate initial data $\left.v_{0}\right)$.

The aim of this paper is to directly solve problem (2.3) when the basic solution $u$ presents a discontinuity through a shock curve. The linearized equation

$$
v_{t}+\left(f^{\prime}(u) v\right)_{x}=0,
$$

although linear, has discontinuous coefficients in this case and does not possess in general a solution in any functional space, this one must be found in a measure space.

3. The solution of the linearized problem when the basic solution is a shock

We will focus our attention on the case of a basic solution $u$ which is discontinuous through a shock curve $\Sigma$ given by

$$
\Sigma=\left\{( x , t ) \in \left[0,+\infty\left[\times\left[t_{0},+\infty[: x=\phi(t)\},\right.\right.\right.\right.
$$

where $\phi$ is a positive $C^{1}$ function defined on some interval $\left[t_{0},+\infty[\subseteq[0,+\infty[\right.$. We suppose that $u$ is smooth outside $\Sigma$ and we make the following assumption:

H1. $\phi$ is a strictly increasing function which satisfies $\phi\left(t_{0}\right)=0$.

This hypothesis means that the discontinuity leaves the boundary at time $t_{0}$ and for $t>t_{0}$ does not cross it again. Let us denote by

$$
D^{-}=\left\{( x , t ) \in \left[0,+\infty\left[\times\left[t_{0},+\infty[: x<\phi(t)\}\right.\right.\right.\right.
$$


and by

$$
D^{+}=\left\{( x , t ) \in \left[0,+\infty\left[\times\left[t_{0},+\infty[: x>\phi(t)\} \cup\left[0,+\infty\left[\times\left[0, t_{0}[\right.\right.\right.\right.\right.\right.\right.
$$

the left and the right sides of $\Sigma$, respectively. Since $u$ is smooth outside $\Sigma$ and of bounded variation over $\left[0,+\infty\left[\times\left[0,+\infty\left[\right.\right.\right.\right.$, we can define for $u_{\left.\right|_{D^{-}}}$and for $u_{\left.\right|_{D^{+}}}$their traces on $\Sigma$. In addition, u satisfies the Rankine-Hugoniot jump condition across $\Sigma$.

Given a bounded variation function $w$ on $\mathbb{R} \times[0,+\infty[$, which is smooth outside the curve $\Sigma$, we will denote by

$$
w^{+}(\phi(t), t)=\lim _{\substack{x \rightarrow \phi(t) \\ x>\phi(t)}} w(x, t) \quad \text { and by } \quad w^{-}(\phi(t), t)=\lim _{\substack{x \rightarrow \phi(t) \\ x<\phi(t)}} w(x, t)
$$

the limits on the right and on the left sides of $\Sigma$, and by

$$
[w](t)=w^{+}(\phi(t), t)-w^{-}(\phi(t), t)
$$

the jump over $\Sigma$. With this notation, the Rankine-Hugoniot jump condition reads as $[f(u)](t)=\phi^{\prime}(t)[u](t)$.

In a general framework, we consider a boundary data $b$ in the space of bounded measures $\mathcal{M}([0,+\infty[)$ of the form

$$
b=\tilde{b}(t)+\bar{b} \delta_{t_{0}},
$$

where $\tilde{b}$ is a bounded variation function over $\left[0,+\infty\left[, \bar{b} \in \mathbb{R}\right.\right.$ and $\delta_{t_{0}}$ is the Dirac measure at $t_{0}$. This kind of data for the linearized problem makes sense if, for instance, the boundary data $a(t)$ for the nonlinear problem is discontinuous at $t_{0}$ and the position of this discontinuity is also perturbed. Let us give the following example: consider boundary data $a$ and its perturbation $a_{\varepsilon}$ given by

$$
a=\left\{\begin{array}{ll}
a^{+}, & t>t_{0}, \\
a^{-}, & t<t_{0},
\end{array} \quad a_{\varepsilon}= \begin{cases}a^{+}+\varepsilon b^{+}, & t>t_{0}+\varepsilon, \\
a^{-}+\varepsilon b^{-}, & t<t_{0}+\varepsilon .\end{cases}\right.
$$

We then obtain that

$$
b=\lim _{\varepsilon \rightarrow 0} \frac{a_{\varepsilon}-a}{\varepsilon}=\left(a^{+}-a^{-}\right) \delta_{t_{0}}+ \begin{cases}b^{+}, & t>t_{0}, \\ b^{-}, & t<t_{0} .\end{cases}
$$

Let us recall the definition of the Dirac measure carried by $\Sigma, \delta_{\Sigma}$, which we will use in the following calculations. The measure $\delta_{\Sigma}$ is defined in the space of locally bounded measures $\mathcal{M}_{l o c}([0,+\infty[\times[0,+\infty[)$ by

$$
<\delta_{\Sigma}, \varphi>=\int_{\Sigma} \varphi d \sigma, \forall \varphi \in C_{0}([0,+\infty[\times[0,+\infty[) .
$$

The main result of this paper establishes that problem (2.3), with boundary data $b$ given by (3.1) and, as basic solution $u$ as described above, possesses a measure solution of the form

$$
\mu=\tilde{v}+\alpha(t) \delta_{\Sigma},
$$

where $\tilde{v}$, the "function part of the measure $v$ ", is a bounded variation function, and where $\alpha(t)$ is a smooth function that we will make precise below. 
Let us parameterize $\Sigma$ by $t \in\left[t_{0},+\infty[\longmapsto(\phi(t), t)\right.$, so that we have

$$
<\delta_{\Sigma}, \varphi>=\int_{t_{0}}^{+\infty} \varphi(\phi(t), t) \sqrt{1+\phi^{\prime 2}(t)} d t .
$$

A solution of equation (2.4) as (3.2) makes sense provided that we define the product of the measure $\delta_{\Sigma}$ by $f^{\prime}(u)$, which is a discontinuous function through $\Sigma$. To do so, we will use the notion of Volpert product, which was introduced in [13], and which we recall here. Let $a: \mathbb{R} \longrightarrow \mathbb{R}$ be a locally summable function. The Volpert superposition is defined by

$$
\widehat{a}(u)(x):= \begin{cases}a(u(x)), & x \in C(u), \\ \int_{0}^{1} a\left(u^{-}(x)+s\left(u^{+}-u^{-}\right)(x)\right) d s, & x \in S(u),\end{cases}
$$

where, for the BV function $u, C(u)$ is the continuity set of $u$ and $S(u)$ is the jump points set of $u$, in such a way that $\mathbb{R} \backslash(C(u) \cup S(u))$ has zero-measure. Let $u \in B V(\mathbb{R})$. Then, if $\widehat{a}(u)$ is summable with respect to the measure $\frac{d u}{d x}$, the Volpert product between $a(u)$ and $\frac{d u}{d x}$ is defined by

$$
a(u) \frac{d u}{d x}:=\widehat{a}(u) \frac{d u}{d x}
$$

$($ see $[13])$.

Now, since we have

$$
<\delta_{\Sigma}, \varphi>=\int_{t_{0}}^{+\infty}<\delta_{\phi(t)}, \varphi(\cdot, t) \sqrt{1+\phi^{\prime 2}(t)}>d t
$$

we define in a natural way the product $f^{\prime}(u) \delta_{\Sigma}$ in such a way that we have in the sense of measures

$$
<f^{\prime}(u) \delta_{\Sigma}, \varphi>=\int_{t_{0}}^{+\infty}<\widehat{f}^{\prime}(u)(\cdot, t) \delta_{\phi(t)}, \varphi(\cdot, t) \sqrt{1+\phi^{\prime 2}(t)}>d t
$$

where $\widehat{f}^{\prime}(u)(x, t)=f^{\prime}(u)(x, t)$, if $x \neq \phi(t)$, and

$$
\widehat{f}^{\prime}(u)(\phi(t), t)=\int_{0}^{1} f^{\prime}\left(u^{-}(\phi(t), t)+s\left(u^{+}-u^{-}\right)(\phi(t), t)\right) d s .
$$

Since we have

$$
\begin{aligned}
& \int_{t_{0}}^{+\infty}<\widehat{f}^{\prime}(u)(\cdot, t) \delta_{\phi(t)}, \varphi(\cdot, t) \sqrt{1+\phi^{\prime 2}(t)}>d t \\
= & \int_{t_{0}}^{+\infty} \varphi(\phi(t), t) \widehat{f}^{\prime}(u)(\phi(t), t) \sqrt{1+\phi^{\prime 2}(t)} d t,
\end{aligned}
$$

we define

$$
f^{\prime}(u) \delta_{\Sigma}:=\widehat{f}^{\prime}(u)(\phi(t), t) \delta_{\Sigma}
$$

It is easy to see that, due to the Rankine-Hugoniot condition through $\Sigma,(3.3)$ becomes $\widehat{f}^{\prime}(u)(\phi(t), t)=\phi^{\prime}(t)$. We can then conclude that $\widehat{f}^{\prime}(u)(\phi(t), t) \delta_{\Sigma}=\phi^{\prime}(t) \delta_{\Sigma}$ and $(3.4)$ implies that $f^{\prime}(u) \delta_{\Sigma}$ is defined in such a way that we have, in $\mathcal{M}_{\text {loc }}([0,+\infty[\times[0,+\infty[)$, 


$$
<f^{\prime}(u) \delta_{\Sigma}, \varphi>:=<\phi^{\prime}(t) \delta_{\Sigma}, \varphi>=\int_{t_{0}}^{+\infty} \phi^{\prime}(t) \varphi(\phi(t), t) \sqrt{1+\phi^{\prime 2}(t)} d t
$$

We begin with a lemma, which states the existence of a classical solution of (2.3) in $D^{-}$and in $D^{+}$. In these sets the linearized equation is a classical linear transport equation, and the classical theory of hyperbolic equations is then applied.

Lemma 3.1. Let $b(t)=\tilde{b}(t) \in B V\left(\left[0,+\infty[)\right.\right.$ and $v_{0}(x) \in B V([0,+\infty[)$ be given. Then the initial and boundary value problem (2.3) has a classical solution $\tilde{v}$ in $\left[0,+\infty\left[\times\left[0,+\infty\left[\backslash \Sigma=D^{-} \cup D^{+}\right.\right.\right.\right.$, such that $\tilde{v}(x, 0)=v_{0}(x)$ and $\tilde{v}(0, t)=\tilde{b}(t)$ for all $t$ such that $f^{\prime}(u(0, t))>0$. We have in addition that $\tilde{v}$ satisfies, in each set $D^{*}=D^{ \pm}$, the weak formulation

$$
\begin{gathered}
\int_{D^{*}}\left(\tilde{v} \varphi_{t}+f^{\prime}(u) \tilde{v} \varphi_{x}\right) d x d t+\int_{0}^{+\infty} f^{\prime}(u(0, t)) \tilde{b}(t) \varphi(0, t) d t \\
+\int_{0}^{+\infty} v_{0}(x) \varphi(x, 0) d x=0
\end{gathered}
$$

for all test functions $\varphi \in C^{1}\left(\left[0,+\infty\left[\times\left[0,+\infty[)\right.\right.\right.\right.$ with compact support in $\overline{D^{*}} \backslash \Sigma$ such that $\varphi(0, t)=0$ if $f^{\prime}(u(0, t)) \leq 0$.

Proof. We let $D^{*}=D^{+}$or $D^{*}=D^{-}$and for simplicity we will use the notation $a(x, t)=f^{\prime}(u(x, t))$. Since $(2.3)$ is a linear transport problem, we can apply the method of characteristics. The characteristic curves $(x(t), t)$ satisfy $\frac{d x}{d t}=a(x(t), t)$, with $x(0)=$ $x_{0}$ or $x(\bar{t})=0$. Since they are the same as for the scalar conservation law $u_{t}+(f(u))_{x}=$ 0 and since Lax entropy conditions $f^{\prime}\left(u^{+}(\phi(t), t)\right)<\phi^{\prime}(t)<f^{\prime}\left(u^{-}(\phi(t), t)\right)$ are satisfied through $\Sigma$, we conclude that characteristics are coming out of the boundary part of $D^{*}$ that corresponds to $\Sigma$. So, we do not have to prescribe any condition in this part of the boundary of $D^{*}$. We can then conclude that there exists a solution $\tilde{v}$ of $(2.3)$ over $D^{*}$ which satisfies

$$
\frac{d}{d t} \tilde{v}(x(t), t)=-\tilde{v}(x(t), t) \frac{\partial a}{\partial x}(x(t), t) .
$$

This solution can then be implicitly defined over the characteristic $\frac{d x}{d t}=a(x, t), x(0)=$ $x_{0}$, by

$$
\tilde{v}(x, t)=v_{0}\left(x_{0}\right)-\int_{0}^{t} \tilde{v}(x(s), s) \frac{\partial a}{\partial x}(x(s), s) d s,
$$

and over the characteristic $\frac{d x}{d t}=a(x, t), x(\bar{t})=0$, by

$$
\tilde{v}(x, t)=\tilde{b}(\bar{t})-\int_{\bar{t}}^{t} \tilde{v}(x(s), s) \frac{\partial a}{\partial x}(x(s), s) d s
$$

where $\bar{t}$ is such that $f^{\prime}(u(0, \bar{t}))>0$. We easily deduce the weak equality (3.6) by integrating by parts the equation $\tilde{v}_{t}+\left(f^{\prime}(u) \tilde{v}\right)_{x}=0$ against a test function $\varphi$ as defined in the lemma.

We can now state the main result of this work. 
TheOREM 3.2. Let $u \in C^{1}([0,+\infty[\times[0,+\infty[\backslash \Sigma)$ be the entropy solution of the boundary and initial value problem (2.1)-(2.2) and let $\tilde{v}$ be the solution of equation (2.3) in $[0,+\infty[\times[0,+\infty[\backslash \Sigma$ given by Lemma 3.1. We assume that hypothesis $H 1$ is satisfied. Then the linearized problem (2.3)-(3.1) has a solution $\mu \in \mathcal{M}_{\text {loc }}([0,+\infty[\times[0,+\infty[) \cap$ $C\left(\left[0, T\left[; \mathcal{M}_{\text {loc }}(\mathbb{R})\right)\right.\right.$, for all $T>0$, given by (3.2), with

$$
\alpha(t)=\frac{\bar{b}-\int_{t_{0}}^{t}\left(\left[f^{\prime}(u) \tilde{v}\right](s)-\phi^{\prime}(s)[\tilde{v}](s)\right) d s}{\sqrt{1+\phi^{\prime 2}(t)}} .
$$

In addition, $\mu$ satisfies

$$
\begin{aligned}
& <\mu_{t}+\left(f^{\prime}(u) \mu\right)_{x}, \varphi> \\
= & \int_{0}^{+\infty} v_{0}(x) \varphi(x, 0) d x+\int_{0}^{+\infty} f^{\prime}(u(0, t)) \tilde{b}(t) \varphi(0, t) d t+<\bar{b} \delta_{t_{0}}, \varphi(0, \cdot)>
\end{aligned}
$$

for all $\varphi \in \mathcal{C}_{0}^{1}\left(\left[0,+\infty\left[\times\left[0,+\infty[)\right.\right.\right.\right.$ such that $\varphi(0, t)=0$ if $f^{\prime}(u(0, t)) \leq 0$.

Proof. Denote respectively by

$$
n_{D^{-}}=\frac{1}{\sqrt{1+\phi^{\prime}(t)^{2}}}\left(1,-\phi^{\prime}(t)\right), \quad n_{D^{+}}=\frac{1}{\sqrt{1+\phi^{\prime}(t)^{2}}}\left(-1, \phi^{\prime}(t)\right)=-n_{D^{-}}
$$

the unit outward normals to $D^{-}$and to $D^{+}$at $(\phi(t), t) \in \Sigma$ for $t>t_{0}$. Let $\varphi \in$ $\mathcal{C}_{0}^{1}([0,+\infty[\times[0,+\infty[)$. We have

$$
\begin{aligned}
<\tilde{v}_{t}+\left(f^{\prime}(u) \tilde{v}\right)_{x}, \varphi> & =-\int_{0}^{+\infty} \int_{0}^{+\infty}\left(\tilde{v} \varphi_{t}+f^{\prime}(u) \tilde{v} \varphi_{x}\right) d x d t \\
& =-\int_{D^{-}}\left(\tilde{v} \varphi_{t}+f^{\prime}(u) \tilde{v} \varphi_{x}\right) d x d t-\int_{D^{+}}\left(\tilde{v} \varphi_{t}+f^{\prime}(u) \tilde{v} \varphi_{x}\right) d x d t
\end{aligned}
$$

We integrate the above equation by parts on $D^{-}$and on $D^{+}$. Since $\tilde{v}$ satisfies $\tilde{v}_{t}+$ $\left(f^{\prime}(u) \tilde{v}\right)_{x}=0$ in each of $D^{ \pm}$, we obtain

$$
\begin{aligned}
& <\tilde{v}_{t}+\left(f^{\prime}(u) \tilde{v}\right)_{x}, \varphi> \\
= & -\int_{\Sigma}\left(\left(f^{\prime}\left(u^{-}\right) \tilde{v}^{-}, \tilde{v}^{-}\right) \cdot n_{D^{-}}+\left(f^{\prime}\left(u^{+}\right) \tilde{v}^{+}, \tilde{v}^{+}\right) \cdot n_{D^{+}}\right) \varphi d s \\
& +\int_{0}^{+\infty} f^{\prime}(u(0, t)) \tilde{v}(0, t) \varphi(0, t) d t+\int_{0}^{+\infty} \tilde{v}(x, 0) \varphi(x, 0) d x \\
= & \int_{t_{0}}^{+\infty}\left(\left(f^{\prime}\left(u^{+}\right) \tilde{v}^{+}-f^{\prime}\left(u^{-}\right) \tilde{v}^{-}\right)-\phi^{\prime}(t)\left(\tilde{v}^{+}-\tilde{v}^{-}\right)\right) \varphi(\phi(t), t) d t \\
& +\int_{0}^{+\infty} f^{\prime}(u(0, t)) \tilde{v}(0, t) \varphi(0, t) d t+\int_{0}^{+\infty} v_{0}(x) \varphi(x, 0) d x \\
= & \int_{t_{0}}^{+\infty}\left(\left[f^{\prime}(u) \tilde{v}\right](t)-\phi^{\prime}(t)[\tilde{v}](t)\right) \varphi(\phi(t), t) d t \\
& +\int_{0}^{+\infty} f^{\prime}(u(0, t)) \tilde{v}(0, t) \varphi(0, t) d t+\int_{0}^{+\infty} v_{0}(x) \varphi(x, 0) d x .
\end{aligned}
$$


We obtain then, in $\mathcal{M}_{l o c}(] 0,+\infty[\times] 0,+\infty[)$,

$$
\tilde{v}_{t}+\left(f^{\prime}(u) \tilde{v}\right)_{x}=\left(\left[f^{\prime}(u) \tilde{v}\right](t)-\phi^{\prime}(t)[\tilde{v}](t)\right)\left(\sqrt{1+\phi^{\prime 2}(t)}\right)^{-1} \delta_{\Sigma} .
$$

Let us define

$$
\beta(t):=\alpha(t) \sqrt{1+\phi^{\prime 2}(t)}=\bar{b}-\int_{t_{0}}^{t}\left(\left[f^{\prime}(u) \tilde{v}\right](s)-\phi^{\prime}(s)[\tilde{v}](s)\right) d s .
$$

We calculate $\left(\alpha(t) \delta_{\Sigma}\right)_{t}+\left(f^{\prime}(u) \alpha(t) \delta_{\Sigma}\right)_{x}$ in the sense of measures, where according to (3.5), we have $f^{\prime}(u) \alpha(t) \delta_{\Sigma}=\phi^{\prime}(t) \alpha(t) \delta_{\Sigma}$. We obtain, for all $\varphi \in C_{0}^{1}([0,+\infty[\times[0,+\infty[)$,

$$
\begin{aligned}
& <\left(\alpha(t) \delta_{\Sigma}\right)_{t}+\left(\phi^{\prime}(t) \alpha(t) \delta_{\Sigma}\right)_{x}, \varphi> \\
= & -<\delta_{\Sigma}, \alpha(t) \varphi_{t}>-<\delta_{\Sigma}, \phi^{\prime}(t) \alpha(t) \varphi_{x}> \\
= & -\int_{t_{0}}^{+\infty}\left[\alpha(s) \varphi_{t}(\phi(s), s)+\phi^{\prime}(s) \alpha(s) \varphi_{x}(\phi(s), s)\right] \sqrt{1+\phi^{\prime 2}(s)} d s \\
= & -\int_{t_{0}}^{+\infty} \beta(s) \frac{d}{d s}(\varphi(\phi(s), s)) d s \\
= & -\int_{t_{0}}^{+\infty} \frac{d}{d s}(\beta(s) \varphi(\phi(s), s)) d s+\int_{t_{0}}^{+\infty} \beta^{\prime}(s) \varphi(\phi(s), s) d s \\
= & \beta\left(t_{0}\right) \varphi\left(0, t_{0}\right)+\int_{t_{0}}^{+\infty} \beta^{\prime}(s) \varphi(\phi(s), s) d s .
\end{aligned}
$$

We have then, in $\mathcal{M}_{l o c}([0,+\infty[\times[0,+\infty[)$,

$$
\left(\alpha(t) \delta_{\Sigma}\right)_{t}+\left(f^{\prime}(u) \alpha(t) \delta_{\Sigma}\right)_{x}=\beta\left(t_{0}\right) \varphi\left(0, t_{0}\right)+\beta^{\prime}(t)\left(\sqrt{1+\phi^{\prime 2}(t)}\right)^{-1} \delta_{\Sigma} .
$$

Now, since we have

$$
\beta^{\prime}(t)=-\left(\left[f^{\prime}(u) \tilde{v}\right](t)-\phi^{\prime}(t)[\tilde{v}](t)\right)
$$

and $\beta\left(t_{0}\right)=\bar{b}$, (3.8) and (3.9) imply that the measure $\mu=\tilde{v}+\alpha(t) \delta_{\Sigma}$ satisfies $\mu_{t}+$ $\left(f^{\prime}(u) \mu\right)_{x}=0$ in $\mathcal{M}_{l o c}(] 0,+\infty[\times] 0,+\infty[)$ and that the weak formulation (3.7) is verified. Finally, since we have

$$
\begin{aligned}
<\alpha(t) \delta_{\Sigma}, \varphi> & =\int_{t_{0}}^{+\infty} \varphi(\phi(t), t) \alpha(t) \sqrt{1+\phi^{\prime 2}(t)} d t \\
& =\int_{t_{0}}^{+\infty}<\alpha(t) \sqrt{1+\phi^{\prime 2}(t)} \delta_{x=\phi(t)}, \varphi(\cdot, t)>d t,
\end{aligned}
$$

if we consider $m=\alpha(t) \delta_{\Sigma}$ and $m(t)=\alpha(t) \sqrt{1+\phi^{\prime 2}(t)} \delta_{\phi(t)}$, by identifying

$$
<m, \varphi>=\int_{t_{0}}^{+\infty}<m(t), \varphi(\cdot, t)>d t,
$$

$\varphi \in C_{0}\left(\left[0,+\infty\left[\times\left[0,+\infty[)\right.\right.\right.\right.$, we obtain that $\alpha(t) \delta_{\Sigma}$ belongs to $C\left(\left[0, T\left[; \mathcal{M}_{l o c}(\mathbb{R})\right)\right.\right.$, and so does $\mu=\tilde{v}+\alpha(t) \delta_{\Sigma}$. This finishes the proof. 
Remark 3.3. Since $\left[f^{\prime}(u) \tilde{v}\right]-\phi^{\prime}(t)[\tilde{v}]$ is in general nonzero, we have that, in general, $\tilde{v}$ is not a weak solution of $v_{t}+\left(f^{\prime}(u) v\right)_{x}=0$ on $] 0,+\infty[\times] 0,+\infty[$.

REMARK 3.4. Since we have assumed $\phi$ to be invertible, we can prove in the same way that the measure solution given by Theorem 3.2 belongs to $C([0,+\infty[; \mathcal{M}([0, T[))$ for all $T>0$.

Remark 3.5. In [12], Poupaud and Rascle introduced, in the framework of a multidimensional transport equation with discontinuous coefficients $a(t, x)$, a definition of measure solution by using the notion of Filippov generalized characteristics, which are the solutions, in a generalized sense, of $\frac{d X}{d t}=a(t, X)$ (see [12]). Their definition requires the uniqueness of these characteristics, which is true if, for instance, the coefficients satisfy a one sided Lipschitz condition, and does not require defining $a$ priori the distributional product between $v$ and the non regular function $a(t, x)$. Nevertheless, they could define this product a posteriori, by using the properties of the Filippov characteristics. Here, we followed an approach in the opposite sense, since we used the notion of Volpert product to define the distributional product between a discontinuous function and a measure, which allowed us to make sense of a solution of the transport equation.

4. The linearization of the entropy condition (2.2) and the boundary condition for the linearized problem in the case of a convex flux $f$

We suppose in this section that the flux function $f$ satisfies $f^{\prime \prime} \geq c$ for some $c>0$. Such a convex function $f$ has an absolute minimum $f\left(u^{*}\right)$ and, for all $a<u^{*}$ (respectively $a>u^{*}$ ), there exists a unique $\bar{a}>u^{*}$ (respectively $\bar{a}<u^{*}$ ) such that $f(a)=f(\bar{a})$. In addition we can easily see that in this case, condition (2.2) becomes

$$
\begin{cases}\left.u(0, t) \in]-\infty, u^{*}\right], & \text { if } a(t) \leq u^{*} \\ u(0, t) \in]-\infty, \overline{a(t)}] \cup\{a(t)\}, & \text { if } a(t) \geq u^{*}\end{cases}
$$

We want to prove that in these conditions, the linearization of the Bardos-Le Roux-Nédélec condition (2.2) leads to a boundary condition for the linearized problem which is consistent with the classical formulation of the boundary condition for a linear transport equation. We suppose from now on that the basic solution $u$ satisfies the two conditions below:

C1. $f^{\prime}(u(0, t)) \neq 0$.

C2. $f^{\prime}(u(0, t)) \neq \overline{a(t)}$, if $a(t)>0$.

These conditions mean that we do not allow the boundary to be characteristic.

Consider then a perturbation $a_{\varepsilon}=a+\varepsilon b_{\varepsilon}$ of the boundary data $a$ with $\left(b_{\varepsilon}\right)_{\varepsilon}$ uniformly bounded in $L^{\infty}$. Suppose that the solution of the perturbed problem can be written as $u_{\varepsilon}=u+\varepsilon v_{\varepsilon}$. Suppose also that for almost all t at least for small $\varepsilon,\left(v_{\varepsilon}(0, t)\right)_{\varepsilon}$ is uniformly bounded in $L^{\infty}$, and that we have $v_{\varepsilon}(0, t) \rightarrow v_{0}(t), b_{\varepsilon}(0, t) \rightarrow b(t)$ a.e. $t>0$ as $\varepsilon \rightarrow 0$. Our purpose is to prove that if $u$ satisfies the Bardos-Le Roux-Nédélec condition (2.2) (or, which is equivalent, condition (4.1)) and if $u_{\varepsilon}(0, t)=u(0, t)+\varepsilon b(0, t)$ satisfies the same condition with respect to $a_{\varepsilon}(t)$, then $v=\lim _{\varepsilon \rightarrow 0} v_{\varepsilon}$ satisfies the boundary condition $v(0, t)=b(t)$ in the classical sense of linear transport equations. We will then prove the following theorem.

TheOREm 4.1. Suppose that there exists $c>0$ such that $f^{\prime \prime} \geq c$ and that conditions 
C1 and C2 hold. Then we have that, for $t>0$, the condition

$$
\left\{\begin{array}{l}
\frac{f(u(0, t))-f(k)}{u(0, t)-k} \leq 0, \text { for all } k \text { between } u(0, t) \text { and } a(t), \\
\frac{f\left(u_{\varepsilon}(0, t)\right)-f(k)}{u_{\varepsilon}(0, t)-k} \leq 0, \text { for all } k \text { between } u_{\varepsilon}(0, t) \text { and } a_{\varepsilon}(t),
\end{array}\right.
$$

implies that $v(0, t)=b(t)$ if $f^{\prime}(u(0, t))>0$ and does not impose any conditions on $v(0, t)$ if $f^{\prime}(u(0, t))<0$.

Proof. Suppose first that $u(0, t)$ is such that $f^{\prime}(u(0, t))>0$. In this case, since $f$ is convex, (2.2) implies $u(0, t)=a(t)$. Since we have $f^{\prime}\left(u_{\varepsilon}(0, t)\right)=f^{\prime}\left(u(0, t)+\varepsilon v_{\varepsilon}(0, t)\right)>0$ for small $\varepsilon$, we obtain $u_{\varepsilon}(0, t)=a_{\varepsilon}(t)$ and therefore $v_{\varepsilon}(0, t)=b_{\varepsilon}(t)$, because we have $u(0, t)=a(t)$. We then obtain $v(0, t)=b(t)$.

Consider now that $f^{\prime}(u(0, t))<0$. We can have three different cases:

1. $a(t)<u^{*}$. In this case, we have $a(t)+\varepsilon b_{\varepsilon}(t)<u^{*}$ and $f^{\prime}\left(u(0, t)+\varepsilon v_{\varepsilon}(0, t)\right)<0$, for small $\varepsilon$, independently of $v_{\varepsilon}(0, t)$. We conclude that $v(0, t)$ is arbitrary. Indeed, condition (4.1) is satisfied independently of $v_{\varepsilon}(0, t)$.

2. $a(t)>u^{*}$. Since we assume condition $\mathrm{C} 2$, we must have $u(0, t)<\overline{a(t)}$ and therefore $u(0, t)+\varepsilon v_{\varepsilon}(0, t)<\overline{a(t)+\varepsilon b_{\varepsilon}(t)}$ for small $\varepsilon$, due to the continuity of the map $a \longmapsto \bar{a}$, and once again independently of the value of $v_{\varepsilon}(0, t)$. We obtain once again that (4.1) is satisfied independently of the value of $v_{\varepsilon}(0, t)$, and thus $v(0, t)$ is then arbitrary.

3. $a(t)=u^{*}$. Since we suppose $\mathrm{C} 1$ and $\mathrm{C} 2$, we have $u(0, t)<u^{*}$. This condition implies the existence of $c<u^{*}$ such that $u(0, t)<c<\overline{a(t)+\varepsilon b_{\varepsilon}(t)}$, for small $\varepsilon$ (note that $\left.\overline{a(t)+\varepsilon b_{\varepsilon}(t)} \underset{\varepsilon \rightarrow 0}{\longrightarrow} \overline{a(t)}=u^{*}\right)$. We can then conclude that $u(0, t)+\varepsilon v_{\varepsilon}(0, t)<c<$ $\overline{a(t)+\varepsilon b_{\varepsilon}(t)}$, for small $\varepsilon$, independently of the value of $v_{\varepsilon}(0, t)$. Once again we have that (4.1) is satisfied independently of the value of $v_{\varepsilon}(0, t)$, and thus $v(0, t)$ is arbitrary.

$\square$

\section{The numerical approximation}

In this section we focus our attention on the numerical approximation of the linearized problem (2.3). We will present a numerical method to approximate the linearized problem, based in a Roe-type scheme which was introduced by Godlewski, Olazabal and Raviart in [6], which we adapt here to the boundary value problem. We will present some numerical tests that illustrate the results of the above sections and at the same time, some numerical results concerning the order of convergence of the numerical scheme. We mention here that in [9] the authors proved the convergence of a class of numerical schemes towards the duality solution of a linear transport equation, in the framework of the Cauchy problem. Although the same analysis is not done here, the numerical results of this section may indicate that we can expect to have a similar result for the initial and boundary value problem (2.3).

We begin by introducing a uniform grid over $[0,+\infty[\times[0,+\infty[$, with time-step $\Delta t$ and mesh-spacing $\Delta x$. The cells are given by $\left[x_{j}, x_{j+1}\left[\times\left[t^{n}, t^{n+1}[\right.\right.\right.$, where we set $x_{j}=j \Delta x, j \in \mathbb{Z}, j \geq 0$, and $t^{n}=n \Delta t, n \in \mathbb{N}$. We also set $\lambda=\frac{\Delta t}{\Delta x}$ and $x_{j+\frac{1}{2}}=\left(j+\frac{1}{2}\right) \Delta x$. We consider now a Roe scheme to approximate the nonlinear boundary and initial 
value problem $(2.1)-(2.2)$. Let $A(u, v)$ be defined by

$$
\left\{\begin{array}{l}
A(u, v)=\frac{f(v)-f(u)}{v-u}, \text { if } v \neq u \\
A(u, u)=f^{\prime}(u)
\end{array}\right.
$$

We set, for $j \geq 1, n \geq 0$,

$$
\begin{gathered}
A_{j}^{n}=A\left(u_{j-\frac{1}{2}}^{n}, u_{j+\frac{1}{2}}^{n}\right), \quad A_{j}^{+n}=\frac{1}{2}\left(A_{j}^{n}+\left|A_{j}^{n}\right|\right), \quad A_{j}^{-n}=\frac{1}{2}\left(A_{j}^{n}-\left|A_{j}^{n}\right|\right), \\
\Delta u_{j}^{n}=u_{j+\frac{1}{2}}^{n}-u_{j-\frac{1}{2}}^{n}
\end{gathered}
$$

and

$$
\begin{gathered}
A_{0}^{n}=A\left(a^{n}, u_{\frac{1}{2}}^{n}\right), \quad A_{0}^{+n}=\frac{1}{2}\left(A_{0}^{n}+\left|A_{0}^{n}\right|\right), \quad A_{0}^{-n}=\frac{1}{2}\left(A_{0}^{n}-\left|A_{0}^{n}\right|\right), \\
\Delta u_{0}^{n}=u_{\frac{1}{2}}^{n}-a^{n} .
\end{gathered}
$$

We consider then a Roe scheme, which can be written as

$$
u_{j+\frac{1}{2}}^{n+1}=u_{j+\frac{1}{2}}^{n}-\frac{\Delta t}{\Delta x}\left(A_{j+1}^{-n} \Delta u_{j+1}^{n}+A^{+}{ }_{j}^{n} \Delta u_{j}^{n}\right), j \geq 0,
$$

with initial and boundary conditions $u_{j+\frac{1}{2}}^{0}$ and $a^{n}$ taken, respectively, to be an approximation of

$$
\frac{1}{\Delta x} \int_{x_{j}}^{x_{j+1}} u_{0}(x) d x, \quad \frac{1}{\Delta t} \int_{t^{n}}^{t^{n+1}} a(t) d t
$$

Notice that (5.1) can also be written in its viscous form

$$
u_{j+\frac{1}{2}}^{n+1}=u_{j+\frac{1}{2}}^{n}-\frac{\Delta t}{\Delta x} \frac{f\left(u_{j+\frac{3}{2}}^{n}\right)-f\left(u_{j-\frac{1}{2}}^{n}\right)}{2}+\frac{Q_{j+1}^{n} \Delta u_{j+1}^{n}-Q_{j}^{n} \Delta u_{j}^{n}}{2},
$$

with numerical viscosity coefficient given by $Q_{j}^{n}=\frac{\Delta t}{\Delta x}\left|A_{j}^{n}\right|$. It is well known that this scheme admits non-entropy solutions which violate stationary shocks. We consider then instead of (5.1) its entropy modification, which is given by (5.2) with

$$
Q_{j}^{n}= \begin{cases}\frac{\Delta t}{\Delta x}\left|A_{j}^{n}\right|, & \text { if }\left|A_{j}^{n}\right| \geq \delta_{j}^{n}, \\ \frac{\Delta t}{\Delta x}\left|\delta_{j}^{n}\right|, & \text { if }\left|A_{j}^{n}\right|<\delta_{j}^{n},\end{cases}
$$

where $\delta_{j}^{n}$ is given by

$$
\sup _{u \in\left[u_{j-\frac{1}{2}}^{n}, u_{j+\frac{1}{2}}^{n}\right]} \max \left\{0, A\left(u, u_{j+\frac{1}{2}}^{n}\right)-A\left(u_{j-\frac{1}{2}}^{n}, u_{j+\frac{1}{2}}^{n}\right), A\left(u_{j-\frac{1}{2}}^{n}, u_{j+\frac{1}{2}}^{n}\right)-A\left(u_{j-\frac{1}{2}}^{n}, u\right)\right\} .
$$

To approximate the linearized problem we will consider the linearized Roe type scheme proposed by Godlewski, Olazabal and Raviart in [6] and [7]. We then set

$$
v_{j+\frac{1}{2}}^{n+1}=v_{j+\frac{1}{2}}^{n}-\frac{\Delta t}{\Delta x}\left(A_{j+1}^{-n} \Delta v_{j+1}^{n}+A_{j}^{+n} \Delta v_{j}^{n}+\left(A_{j+1}^{n}-A_{j}^{n}\right) v_{j+\frac{1}{2}}^{n}\right),
$$


with initial and boundary conditions given to be an approximation of

$$
\frac{1}{\Delta x} \int_{x_{j}}^{x_{j+1}} v_{0}(x) d x, \quad \frac{1}{\Delta t} \int_{t^{n}}^{t^{n+1}} b(t) d t
$$

Notice then this scheme corresponds to an upwind discretization of equation (2.4). We will suppose from now on the following CFL condition:

$$
\frac{\Delta t}{\Delta x} \max \left\{\sup _{x \geq 0}\left|f^{\prime}\left(u_{0}(x)\right)\right|, \sup _{t \geq 0}\left|f^{\prime}(a(t))\right|\right\} \leq 1 .
$$

In the following, we present some results obtained for Burgers' equation $(f(u)=$ $\frac{u^{2}}{2}$ ), in the setting of a Riemann type problem, i.e., with constant data $u_{0}, v_{0}, a$, and $b$. We recall that, in this case, in [5] Dubois and LeFloch proved that the solution of problem (2.1) is the restriction to $[0,+\infty[\times[0,+\infty[$ of the solution of the Riemann problem for the scalar equation with initial data

$$
\begin{cases}a, & x<0 \\ b, & x>0\end{cases}
$$

In the particular case of Burgers' equation, the solution of this Riemann problem is either a shock wave connecting the states $a$ and $u_{0}$, which propagates with speed $\sigma=\frac{a+u_{0}}{2}$, if $a>u_{0}$, or a rarefaction wave if $a<u_{0}$.

First, we will focus our attention in the case where the basic solution is a shock propagating with positive speed, which corresponds to the case studied in the previous sections. Secondly, we will analyze the cases of a stationary shock and a rarefaction wave. In this last case, the solution of the linearized problem can be obtained directly, and we consider it here to illustrate the numerical results of convergence that we obtained. The spatial domain that we consider for the numerical tests is the interval $[0,1]$, and the time $t$ is such that the wave has not yet reached the boundary $x=1$.

5.1. The case of a shock propagating with positive speed. The basic solution $u$ is the shock wave

$$
u(x, t)= \begin{cases}a, & x<\sigma t \\ u_{0}, & x>\sigma t\end{cases}
$$

which propagates with speed $\sigma=\frac{u_{0}+a}{2}>0$. Let the boundary condition $b$ and the initial condition $v_{0}$ for the linearized problem be given. Then, the solution of the linearized problem given by Theorem 3.2 is $\tilde{v}+\alpha(t) \delta_{x=\sigma t}$, with

$$
\tilde{v}=\left\{\begin{array}{ll}
b, & x<\sigma t, \\
v_{0}, & x>\sigma t
\end{array} \quad \text { and } \quad \alpha(t)=\left[-\left(u_{0} v_{0}-a b\right)+\frac{u_{0}+a}{2}\left(v_{0}-b\right)\right] t .\right.
$$

We present in the following figures the numerical results obtained for the data $u_{0}=$ $-1, a=2, v_{0}=10$ and $b=1$, in such a way that the linearized solution is a Dirac measure concentrated on $x=\frac{1}{2} t$. We considered here a CFL value of 0.5 .

We can see that the numerical solution approaches a Dirac mass concentrated on $x=0.25$ at time $t=0.5$. If we denote by $v_{\Delta}$ the numerical solution, the table below proves that $\left\|\tilde{v}-v_{\Delta}\right\|_{L^{1}} \approx 8.25$, where $\tilde{v}$ is the classical solution given by Lemma 3.1 

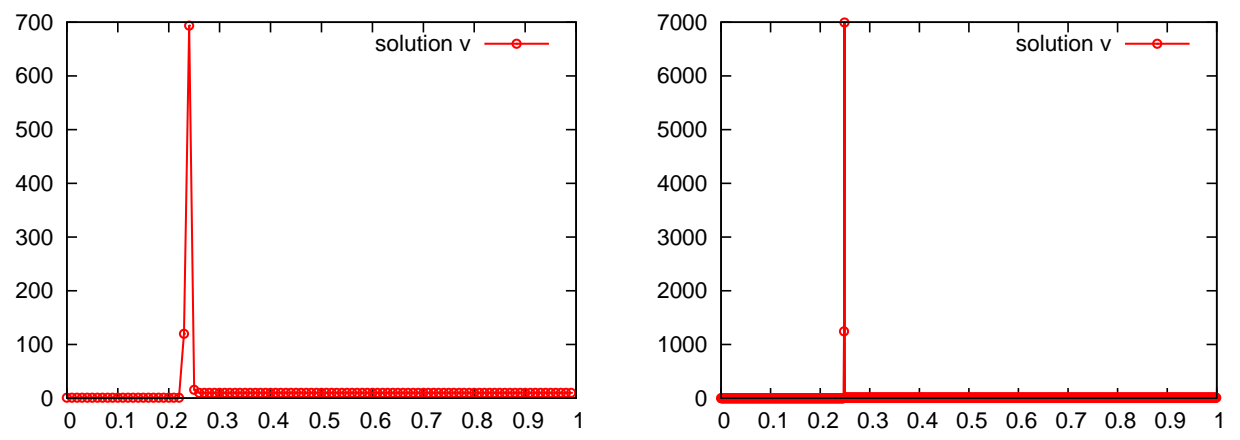

FIGURE 5.1. Linearized solution at time $t=0.5$ with $\Delta x=0.01$ (on the left) and $\Delta x=0.001$ (on the right).

and $\alpha(0,5)=8.25$, where 8.25 is exactly the value of the Dirac coefficient $\alpha(t)$ at $t=0.5$.

\begin{tabular}{c||c|c|c|c|c|c}
\hline$\Delta x$ & 0.01 & 0.0033 & 0.001 & 0.00033 & 0.0001 & 0.000033 \\
\hline$\left\|\tilde{v}-v_{\Delta}\right\|_{L^{1}}$ & 8.1709 & 8.2236 & 8.2421 & 8.2474 & 8.2492 & 8.2497 \\
\hline
\end{tabular}

The next figure presents an error curve, shown in logarithmic scale, between the exact solution and the numerical solution. We propose to measure this error by calculating $E_{\Delta}=\left|\left\|v_{\Delta}-\tilde{v}\right\|_{L^{1}}-\alpha(0.5)\right|$.

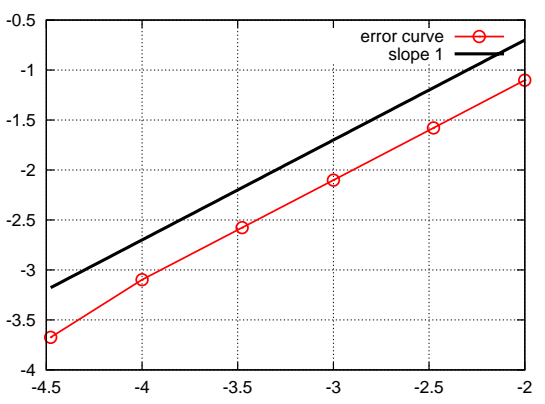

FiguRE 5.2. Error between exact and approximated solution, as a function of mesh spacing, shown in logarithmic scale. The $x$-coordinate is $\log (\Delta x)$ and the $y$-coordinate is $\log \left(E_{\Delta}\right)$.

REMARK 5.1. We obtain the same values no matter we consider $E_{\Delta}$ or $\left\|v_{\Delta}-\tilde{v}\right\|_{L^{1}([0.1] \backslash[0.25-3 \Delta x, 0.25+3 \Delta x])}+\left|\left\|v_{\Delta}-\tilde{v}\right\|_{([0.25-3 \Delta x, 0.25+3 \Delta x])}-\alpha(0.5)\right|$ to measure the error between exact and approximated solution.

5.2. The case of a stationary shock. We consider now the case where the basic solution is a stationary shock, more precisely that the discontinuity arises at the boundary $x=0$. Therefore, hypothesis H1 of section 3 is not satisfied. From a numerical point of view, a Dirac mass appears at $x=0$. This result was expected, even though we did not study this case earlier. 


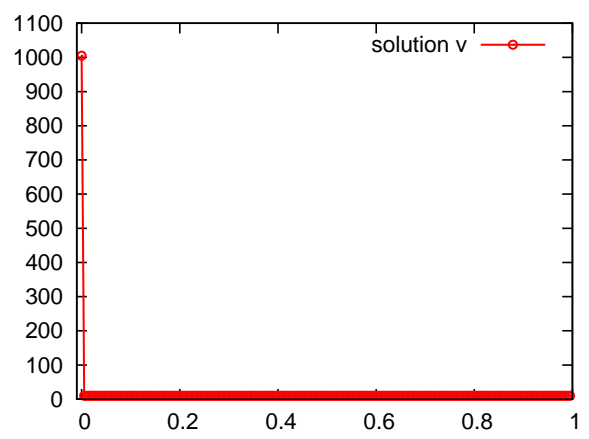

FIGURE 5.3. Linearized solution at time $t=0.5$ when basic solution is a stationary shock.

5.3. The case of a rarefaction wave. We consider in this paragraph that the basic solution is the rarefaction wave

$$
u(x, t)= \begin{cases}a, & \frac{x}{t} \leq a \\ \frac{x}{t}, & a \leq \frac{x}{t} \leq u_{0}, \\ u_{0}, & \frac{x}{t} \geq u_{0} .\end{cases}
$$

In this case, we can directly solve the linearized problem. Indeed, let us look for a self-similar solution of (2.3). Denote by $v\left(\frac{x}{t}\right)$ such a solution. We have then that $v$ satisfies the advection equation $v_{t}+a v_{x}=0$ for $\frac{x}{t}<a$, and we get $v\left(\frac{x}{t}\right)=b$ if $\frac{x}{t}<a$. In the same way we get $v\left(\frac{x}{t}\right)=v_{0}$ if $\frac{x}{t}>u_{0}$. Now we have that in $\left\{(x, t): a<\frac{x}{t}<u_{0}\right\}, v$ satisfies

$$
v_{t}+\left(\frac{x}{t} v\right)_{x}=0
$$

If we put $\xi=\frac{x}{t}$, the above equation reads

$$
-\frac{\xi}{t} v^{\prime}(\xi)+\frac{1}{t} v+\frac{\xi}{t} v^{\prime}(\xi)=0
$$

i.e., $\frac{1}{t} v=0$. We obtain $v\left(\frac{x}{t}\right)=0$, for all $(x, t)$ such that $a<\frac{x}{t}<u_{0}$. The solution of the linearized problem is therefore given by

$$
v(x, t)= \begin{cases}b, & \frac{x}{t} \leq a \\ 0, & a \leq x \\ v_{0}, & \frac{x}{t} \geq u_{0} .\end{cases}
$$

We present below the numerical results that we obtained with the following data: $a=1, u_{0}=2, b=4$ and $v_{0}=10$, taken in such a way that the left side of the rarefaction $u$ propagates with positive speed. Once again CFL is taken to be 0.5 .

Figure 5.5 represents the error between the exact and the approximated solution in the $L^{1}$ norm, as a function of mesh-spacing.

We finally analyze the case where the basic solution $u$ is a sonic rarefaction. In this case, the basic solution and the linearized solution are given by

$$
u(x, t)=\left\{\begin{array}{ll}
\frac{x}{t}, & 0 \leq \frac{x}{t} \leq u_{0}, \\
u_{0}, & \frac{x}{t} \geq u_{0},
\end{array} \quad v(x, t)= \begin{cases}0, & 0 \leq \frac{x}{t} \leq u_{0}, \\
v_{0}, & \frac{x}{t} \geq u_{0},\end{cases}\right.
$$



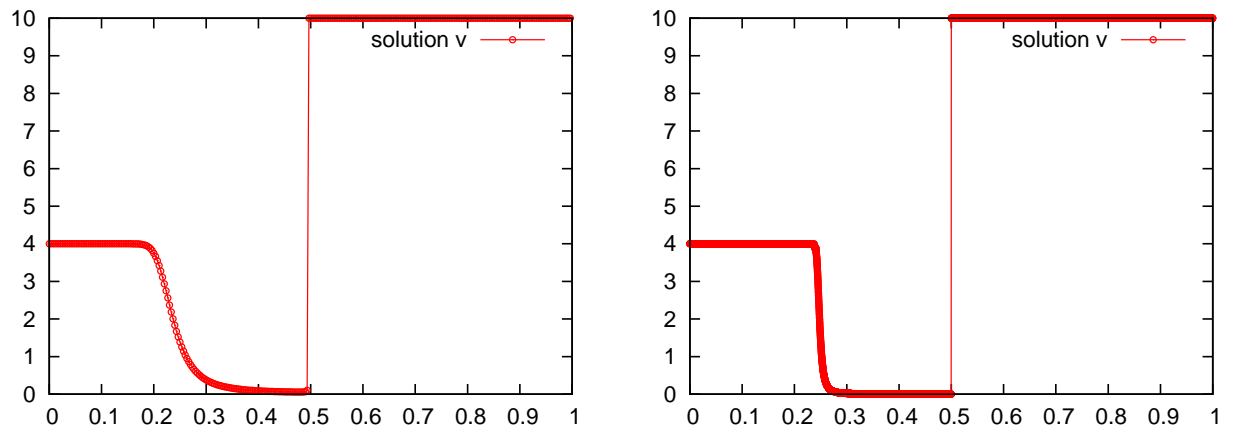

Figure 5.4. Linearized solution at time $t=0.25$ with $\Delta x=0.0033$ (on the left) and $\Delta x=10^{-5}$ (on the right).

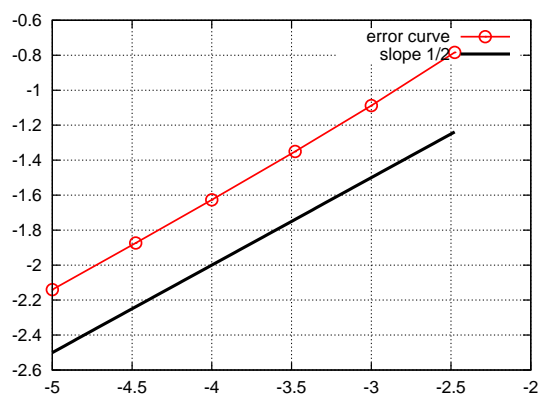

FiguRE 5.5. Error between exact and approximated solution in $L^{1}$.

respectively. We present the numerical results obtained with data $u_{0}, b$, and $v_{0}$ taken to be the same as in the above example and for $a=-1$ and $a=0$.

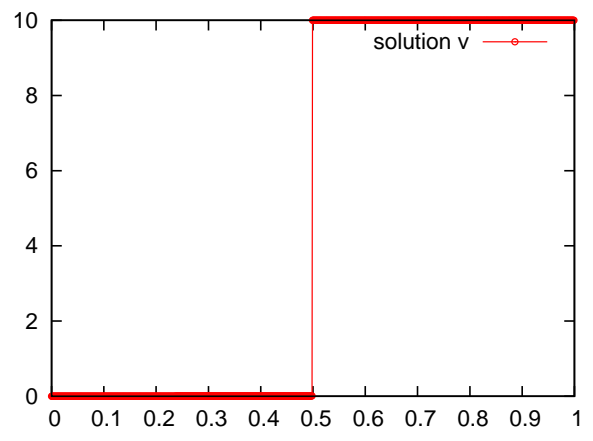

Figure 5.6. $a=-1$ : Linearized solution at time $t=0.5$.

We conjecture that the numerical solution given by the scheme described in this section approaches the exact solution, which equals 0 if $x<0.5$ and 10 if $x>0.5$.

REMARK 5.2. If we consider $u$ to be a shock or a rarefaction solution of Burgers' 

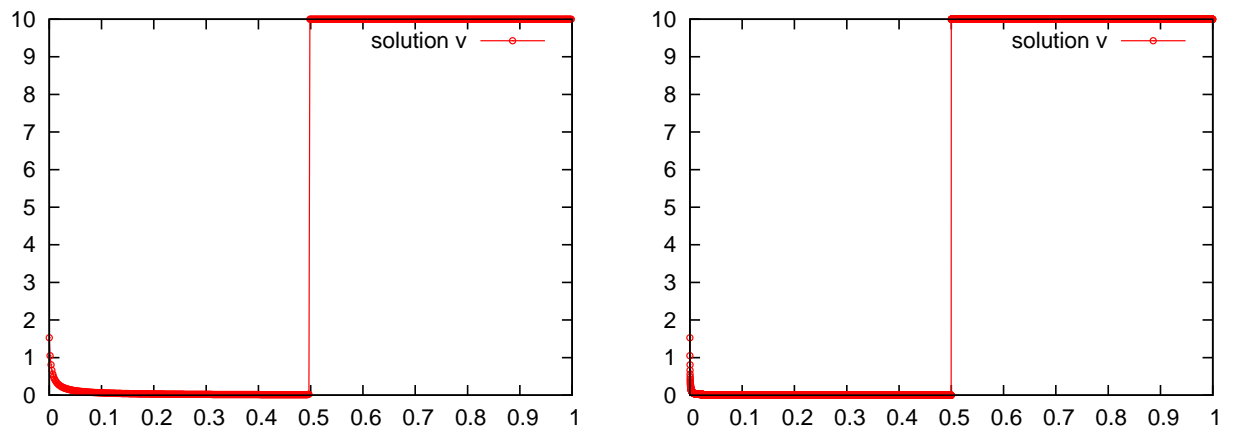

Figure 5.7. $a=0$ : Linearized solution at time $t=0.5$, for $\Delta x=0.0033$ (on the left) and $\Delta x=$ $10^{-5}$ (on the right).

equation, in [12] the authors proved that the measure solution of the equation $v_{t}+$ $\left(\frac{u}{2} v\right)_{x}=0$ is $u$ itself, as expected. If we replace $u$ by $\frac{u}{2}$ in the numerical scheme (5.3), we can see that we recover a Roe discretization of Burgers' equation. Hence, numerically we also recover $u$ as the solution of the above equation, as it was predicted in $[12]$.

Acknowledgements. The author would like to thank P.-A. Raviart for bringing this problem to her attention. She also thanks him and E. Godlewski for many helpful discussions.

\section{REFERENCES}

[1] C. Bardos, A. Le Roux and J.C. Nédélec, First order quasilinear equations with boundary conditions, Comm. Partial Differential Equations, 4(9), 1017-1034, 1979.

[2] C. Bardos and O. Pironneau, A formalism for the differentiation of conservation laws, C.R. Acad. Sci. Paris, Ser. I, 335, 839-845, 2002.

[3] F. Bouchut and F. James, One-dimensional transport equations with discontinuous coefficients, Nonlinear Anal., 32, 891-933, 1998.

[4] F. Bouchut and F. James, Differentiability with respect to the initial data for a scalar conservation law, Proceedings of the Seventh International Conference on Hyperbolic Problems, Zürich, 113-118, 1998.

[5] F. Dubois and P.G. LeFloch, Boundary conditions for nonlinear hyperbolic problems of conservation laws, J. Differential Equations, 71, 93-122, 1988.

[6] E. Godlewski, M. Olazabal and P.A. Raviart, On the linearization of hyperbolic systems of conservation laws, application to stability, Équations aux dérivées partielles et applications, articles dédiés à J.L. Lions, Gauthier-Villars, Paris, 549-570, 1998.

[7] E. Godlewski, M. Olazabal and P.A. Raviart, On the linearization of systems of conservation laws for fluids at a material contact discontinuity, J. Math. Pures Appl., 78, 1013-1042, 1999.

[8] E. Godlewski and P.A. Raviart, The linearized stability of solutions of nonlinear hyperbolic systems of conservation laws, a general numerical approach, Math. Comput. Simulation, 50, 77-95, 1999.

[9] L. Gosse and F. James, Numerical approximations of one-dimensional linear conservation equations with discontinuous coefficients, Math. Comput., 69, 987-1015, 2000.

[10] A. Majda, The stability of multidimensional shock fronts, Memoirs of the AMS, 275, Amer. Math. Soc., Providence, RI, 1983.

[11] A. Majda, The existence of multidimensional shock fronts, Memoirs of the AMS, 281, Amer. Math. Soc., Providence, RI, 1983. 
[12] F. Poupaud and M. Rascle, Measure solutions to the linear multi-dimensional transport equation with non-smooth coefficients, Comm. Partial Differential Equations, 22, 337-358, 1997.

[13] A. Volpert, The spaces BV and quasilinear equations, Math. USSR-Sb., 2, 225-267, 1967. 